\title{
A Comparison Theorem for Oscillation of the Even-Order Nonlinear Neutral Difference Equation
}

\author{
Quanxin Zhang \\ Department of Mathematics, Binzhou University, Binzhou, Shandong 256603, China \\ Correspondence should be addressed to Quanxin Zhang; 3314744@163.com
}

Received 9 December 2013; Accepted 28 March 2014; Published 10 April 2014

Academic Editor: Zong-Xuan Chen

Copyright (C) 2014 Quanxin Zhang. This is an open access article distributed under the Creative Commons Attribution License, which permits unrestricted use, distribution, and reproduction in any medium, provided the original work is properly cited.

\begin{abstract}
A comparison theorem on oscillation behavior is firstly established for a class of even-order nonlinear neutral delay difference equations. By using the obtained comparison theorem, two oscillation criteria are derived for the class of even-order nonlinear neutral delay difference equations. Two examples are given to show the effectiveness of the obtained results.
\end{abstract}

\section{Introduction}

Recently there have been a lot of research papers in connection with the oscillation of solutions of difference equations with or without neutral terms. The literature on the oscillation of neutral delay difference equations is growing very fast, and it can be widely applied to the reality. In fact, neutral delay difference equations arise in modelling of the networks containing lossless transmission lines (as in high speed computers where the lossless transmission lines are used to interconnect switching circuits). For recent contributions regarding the theoretical part and providing systematic treatment of oscillation of solutions of neutral type difference equations, the readers can refer to the recent monographs by Agarwal [1], Györi and Ladas [2].

The oscillation behavior of the even-order nonlinear neutral differential equation

$$
[x(t)+p(t) x(\tau(t))]^{(n)}+q(t) f[x(\sigma(t))]=0
$$

has been established by Zhang et al. [3]. In this paper, the discrete analogue of the above equation is considered. We consider the even-order nonlinear neutral difference equation,

$$
\Delta^{m}\left(x_{n}+p_{n} x_{n-\tau}\right)+q_{n} f\left(x_{n-k}\right)=0,
$$

where $m \geq 2$ is an even and $\tau, k \in \mathbb{N}$; let $\mathbb{N}$ denote the set of all natural numbers; $n \in N\left(n_{0}\right)=\left\{n_{0}, n_{0}+1, n_{0}+2, \ldots\right\}$; $n_{0}$ is a nonnegative integer; $\Delta$ denotes the forward difference operator defined by $\Delta x_{n}=x_{n+1}-x_{n}, \Delta^{m} x_{n}=\Delta^{m-1} x_{n+1}-$ $\Delta^{m-1} x_{n}$.

Throughout this paper, the following conditions are assumed to hold:

$\left(\mathrm{H}_{1}\right)\left\{p_{n}\right\}$ is a sequence of nonnegative real number, $0 \leq$ $p_{n}<1$, and $\left\{q_{n}\right\}$ is a sequence of nonnegative real number with $\left\{q_{n}\right\}$ being not eventually identically equal to zero;

$\left(\mathrm{H}_{2}\right) f: \mathbb{R} \rightarrow \mathbb{R}(\mathbb{R}=(-\infty,+\infty))$ is a continuous odd function, and $x f(x)>0$ for all $x \neq 0$.

Before deriving the main results, the following definitions are given.

Definition 1. By a solution of (2), one means a real sequence $\left\{x_{n}\right\}$ defined for $n \geq n_{0}-\theta(\theta=\max \{\tau, k\})$ which satisfies (2) for $n \in N\left(n_{0}\right)$.

In this paper, we restrict our attention to nontrivial solutions of (2).

Definition 2. A nontrivial solution $\left\{x_{n}\right\}$ of (2) is said to be oscillatory if the terms $x_{n}$ of the sequence are neither eventually positive nor eventually negative. Otherwise, it is called nonoscillatory.

Definition 3. An equation is said to be oscillatory if all its solutions are oscillatory. 
In 2004, Stavroulakis [4] studied the oscillatory behavior of all solutions of first-order delay difference equation,

$$
x_{n+1}-x_{n}+p_{n} x_{n-k}=0,
$$

and established one new oscillation criterion. Thandapani et al. [5] studied the oscillatory behavior of all solutions of second-order neutral delay difference equation,

$$
\Delta^{2}\left(y_{n}-p y_{n-k}\right)-q_{n} f\left(y_{n-t}\right)=0,
$$

and established a number of new oscillation criteria. In 2000, Zhou et al. [6] studied the oscillatory behavior of all solutions of even-order neutral delay difference equation,

$$
\Delta^{m}\left(x_{n}-p_{n} g\left(x_{n-k}\right)\right)-q_{n} h\left(x_{n-l}\right)=0,
$$

and established three new oscillation criteria under certain conditions. The studies on oscillatory behavior of all solutions of even-order delay difference equations, we recommend referring to [7-10]. On the basis of the above work, we studied the oscillatory behavior of all solutions of (2). Firstly, a comparison theorem on oscillation behavior is established for (2). The comparison theorem changes the discriminant criteria of the oscillation of (2) into the oscillation's discriminant criteria in the first-order nonneutral delay difference equations. Then, by using the above comparison theorem, we obtain some oscillation criteria for (2) and improve the well-known results of Ladas et al. [11], Erbe and Zhang [12], and Stavroulakis [4]. In particular, the results are new when $m=2, p_{n} \equiv 0$.

The paper is organized as follows. In Section 2, a comparison theorem on oscillation behavior is firstly established for a class of even-order nonlinear neutral delay difference equations. Then the comparison theorem changes the discriminant of the oscillation in the even-order nonlinear neutral delay difference equation into the oscillation's discriminant in the first-order nonneutral delay difference equations. In Section 3, some oscillation criteria are obtained for the class of even-order nonlinear neutral delay difference equation by using the above comparison theorem. In Section 4, two examples are given.

\section{Comparison Theorem}

To obtain the comparison theorem in this section, we need the following lemmas which can be founded in [1]; see also Chen [7] and Thandapani and Arul [8].

Lemma 4. Let $\left\{u_{n}\right\}$ be a sequence of real numbers for $n \geq n_{0}$. Let $\left\{u_{n}\right\}$ and $\left\{\Delta^{m} u_{n}\right\}$ be of constant sign, where $\Delta^{m} u_{n}$ is not identically zero for $n \geq n_{1}$. If

$$
u_{n} \Delta^{m} u_{n} \leq 0
$$

then

(i) there is a natural number $n_{2} \geq n_{1}$ such that the sequences $\left\{\Delta^{j} u_{n}, j=1,2, \ldots, m-1\right\}$ are of constant sign for $n \geq n_{2}$; (ii) there exists a number $l \in\{0,1, \ldots, m-1\}$ with $(-1)^{m-l-1}=1$ such that

$$
\begin{gathered}
u_{n} \Delta^{j} u_{n}>0 \quad \text { for } j=0,1,2, \ldots, l, n \geq n_{2}, \\
(-1)^{j-l} u_{n} \Delta^{j} u_{n}>0 \quad \text { for } j=l+1, \ldots, m-1, n \geq n_{2} .
\end{gathered}
$$

Lemma 5. Observe that under the hypotheses of Lemma 4, if $\left\{u_{n}\right\}$ is increasing for $n \geq n_{0}$, then there exists a natural number $n_{1} \geq n_{0}$ such that, for all $n \geq 2^{m-1} n_{1}$,

$$
u_{n} \geq \frac{\lambda_{m}}{(m-1) !} n^{m-1} \Delta^{m-1} u_{n}
$$

where $\lambda_{m}=1 / 2^{(m-1)^{2}}$.

Theorem 6. Assume that conditions $\left(H_{1}\right)$ and $\left(H_{2}\right)$ hold. Let $|f(x)| \geq|x|$ for all $|x| \geq x_{0}>0$. If there exists a constant $\lambda_{m}=1 / 2^{(m-1)^{2}}$, such that the first-order difference equation

$$
\Delta z_{n}+\frac{\lambda_{m}}{(m-1) !} q_{n}(n-k)^{m-1}\left(1-p_{n-k}\right) z_{n-k}=0
$$

is oscillatory, then (2) is oscillatory.

Proof. Suppose that (2) has a nonoscillatory solution $\left\{x_{n}\right\}$. Without the loss of generality, we assume that $\left\{x_{n}\right\}$ is an eventually positive solution of (2); then there is a natural number $n_{1} \geq n_{0}$ such that $x_{n}>0, x_{n-k}>0, x_{n-\tau}>0$, and $x_{n-k-\tau}>0$ for all $n \geq n_{1}$. Let

$$
y_{n}=x_{n}+p_{n} x_{n-\tau} .
$$

Then, from $\left(\mathrm{H}_{1}\right)$ and $\left(\mathrm{H}_{2}\right)$, there exists a natural number $n_{2} \geq$ $n_{1}$ such that

$$
y_{n}>0, \quad \Delta^{m} y_{n} \leq 0 \quad \forall n \geq n_{2} .
$$

By Lemma 4 , there exist an integer $n_{3} \geq n_{2}$ and an integer $l(0 \leq l \leq m)$, where $(m+l)$ is an odd integer. For all $n \geq n_{3}$, we can get

$$
\begin{gathered}
\Delta^{j} y_{n}>0 \text { for } j=1,2, \ldots, l \\
(-1)^{(j-l)} \Delta^{j} y_{n}>0 \text { for } j=l+1, \ldots, m-1 .
\end{gathered}
$$

Thus from (12), $\Delta y_{n}>0$ and $\Delta^{m-1} y_{n}>0$ for $n \geq n_{3}$. By Lemma 5 , there exists an integer $n_{4} \geq n_{3}$. For all $n \geq 2^{m-1} n_{4}$, we derive

$$
y_{n} \geq \frac{\lambda_{m}}{(m-1) !} n^{m-1} \Delta^{m-1} y_{n}, \quad \lambda_{m}=\frac{1}{2^{(m-1)^{2}}} .
$$

From (10),

$$
x_{n-k}=y_{n-k}-p_{n-k} x_{n-k-\tau} .
$$

Consequently, we have

$$
\Delta^{m} y_{n}+q_{n} f\left(y_{n-k}-p_{n-k} x_{n-k-\tau}\right)=0,
$$


Noting that $|f(x)| \geq|x|$, for all $|x| \geq x_{0}>0$, we obtain

$$
\Delta^{m} y_{n}+q_{n}\left(y_{n-k}-p_{n-k} x_{n-k-\tau}\right) \leq 0,
$$

for all sufficient large $n$.

By $y_{n} \geq x_{n}, \Delta y_{n}>0$, and $n-k-\tau \leq n-k$, we obtain

$$
\Delta^{m} y_{n}+q_{n}\left(y_{n-k}-p_{n-k} x_{n-k-\tau}\right) \geq \Delta^{m} y_{n}+q_{n}\left(1-p_{n-k}\right) y_{n-k} .
$$

Therefore, we have

$$
\begin{array}{r}
\Delta^{m} y_{n}+q_{n}\left(1-p_{n-k}\right) y_{n-k} \leq 0, \\
\text { for all sufficient large } n .
\end{array}
$$

Now, by using (13), we have that for $\lambda_{m}=1 / 2^{(m-1)^{2}}$,

$$
y_{n-k} \geq \frac{\lambda_{m}}{(m-1) !}(n-k)^{m-1} \Delta^{m-1} y_{n-k}
$$

for all sufficient large $n$.

Thus, we get

$$
\Delta^{m} y_{n}+\frac{\lambda_{m}}{(m-1) !} q_{n}(n-k)^{m-1}\left(1-p_{n-k}\right) \Delta^{m-1} y_{n-k} \leq 0,
$$

for all sufficient large $n$,

where $\lambda_{m}=1 / 2^{(m-1)^{2}}$. Let $u_{n}=\Delta^{m-1} y_{n}$; then for large enough $n$, we get

$$
\Delta u_{n}+\frac{\lambda_{m}}{(m-1) !} q_{n}(n-k)^{m-1}\left(1-p_{n-k}\right) u_{n-k} \leq 0
$$

where $\lambda_{m}=1 / 2^{(m-1)^{2}}$. Therefore, inequality (21) has an eventually positive solution. By Lemma 5 in [9], (9) has an eventually positive solution which contradicts that (9) is oscillatory. This completes the proof.

\section{Applications of the Comparison Theorem}

The following lemma is well known (see, e.g., $[2,11,12]$ and the references therein).

Lemma 7. Let $\left\{q_{n}\right\}$ be a sequence of eventually nonnegative real number and $k \geq 1$; if either

$$
\liminf _{n \rightarrow \infty} \sum_{i=n-k}^{n-1} q_{i}>\left(\frac{k}{1+k}\right)^{1+k}
$$

or

$$
\limsup _{n \rightarrow \infty} \sum_{i=n-k}^{n} q_{i}>1,
$$

then the first-order difference equation

$$
\Delta x_{n}+q_{n} x_{n-k}=0
$$

is oscillatory.
Thus, from Theorem 6 and Lemma 7, we can obtain the following results.

Theorem 8. Assume that conditions $\left(H_{1}\right)$ and $\left(H_{2}\right)$ hold. Let $|f(x)| \geq|x|$ for all $|x| \geq x_{0}>0$. For $k \geq 1$, if either

$$
\begin{gathered}
\liminf _{n \rightarrow \infty} \sum_{i=n-k}^{n-1} q_{i}(i-k)^{m-1}\left(1-p_{i-k}\right) \\
>2^{(m-1)^{2}}(m-1) !\left(\frac{k}{1+k}\right)^{1+k}
\end{gathered}
$$

or

$$
\limsup _{n \rightarrow \infty} \sum_{i=n-k}^{n} q_{i}(i-k)^{m-1}\left(1-p_{i-k}\right)>2^{(m-1)^{2}}(m-1) !,
$$

then (2) is oscillatory.

Proof. From (25) and (26), we can obtain

$$
\liminf _{n \rightarrow \infty} \sum_{i=n-k}^{n-1} \frac{\lambda_{m}}{(m-1) !} q_{i}(i-k)^{m-1}\left(1-p_{i-k}\right)>\left(\frac{k}{1+k}\right)^{1+k}
$$

or

$$
\limsup _{n \rightarrow \infty} \sum_{i=n-k}^{n} \frac{\lambda_{m}}{(m-1) !} q_{i}(i-k)^{m-1}\left(1-p_{i-k}\right)>1,
$$

where $\lambda_{m}=1 / 2^{(m-1)^{2}}$. By Lemma 7 , we know (9) is oscillatory. Then similar to the proof of Theorem 6 , the results follow immediately. This completes the proof.

According to Theorem 8, we obtain Corollary 9.

Corollary 9. Assume that conditions $\left(H_{1}\right)$ and $\left(H_{2}\right)$ hold. Let $|f(x)| \geq|x|$ for all $|x| \geq x_{0}>0$. For $k \geq 1$, when $p_{n} \equiv 0$, $m=2$, if either

$$
\liminf _{n \rightarrow \infty} \sum_{i=n-k}^{n-1} q_{i}(i-k)>2\left(\frac{k}{1+k}\right)^{1+k}
$$

or

$$
\limsup _{n \rightarrow \infty} \sum_{i=n-k}^{n} q_{i}(i-k)>2,
$$

then the second-order difference equation

$$
\Delta^{2} x_{n}+q_{n} f\left(x_{n-k}\right)=0
$$

is oscillatory.

The following lemma is given in [4, Theorem 2.6].

Lemma 10. Let $\left\{q_{n}\right\}$ be a sequence of nonnegative real numbers and $k$ a positive integer. Assume that

$$
0<\alpha \leq\left(\frac{k}{1+k}\right)^{1+k}
$$


if either

$$
\limsup _{n \rightarrow \infty} \sum_{i=n-k}^{n-1} q_{i}>1-\frac{\alpha^{2}}{4}
$$

or

$$
\limsup _{n \rightarrow \infty} \sum_{i=n-k}^{n-1} q_{i}>1-\alpha^{k}
$$

then (24) is oscillatory.

Thus, from Theorem 6 and Lemma 10, we can obtain the following results.

Theorem 11. Assume that conditions $\left(H_{1}\right)$ and $\left(H_{2}\right)$ hold. Let $|f(x)| \geq|x|$ for all $|x| \geq x_{0}>0$ and let $k$ be a positive integer. Assume that

$$
0<\alpha \leq\left(\frac{k}{1+k}\right)^{1+k}
$$

if either

$$
\begin{gathered}
\limsup _{n \rightarrow \infty} \sum_{i=n-k}^{n-1} q_{i}(i-k)^{m-1}\left(1-p_{i-k}\right) \\
>2^{(m-1)^{2}}(m-1) !\left(1-\frac{\alpha^{2}}{4}\right)
\end{gathered}
$$

or

$$
\begin{gathered}
\limsup _{n \rightarrow \infty} \sum_{i=n-k}^{n-1} q_{i}(i-k)^{m-1}\left(1-p_{i-k}\right) \\
>2^{(m-1)^{2}}(m-1) !\left(1-\alpha^{k}\right)
\end{gathered}
$$

then (2) is oscillatory.

Proof. From (36) and (37), we can obtain

$$
\limsup _{n \rightarrow \infty} \sum_{i=n-k}^{n-1} \frac{\lambda_{m}}{(m-1) !} q_{i}(i-k)^{m-1}\left(1-p_{i-k}\right)>1-\frac{\alpha^{2}}{4}
$$

or

$$
\limsup _{n \rightarrow \infty} \sum_{i=n-k}^{n-1} \frac{\lambda_{m}}{(m-1) !} q_{i}(i-k)^{m-1}\left(1-p_{i-k}\right)>1-\alpha^{k}
$$

where $\lambda_{m}=1 / 2^{(m-1)^{2}}$. By Lemma 10, we know (9) is oscillatory. Then similar to the proof of Theorem 6 , the results follow immediately. This completes the proof.

According to Theorem 11, we can obtain the following corollary.

Corollary 12. Assume that conditions $\left(H_{1}\right)$ and $\left(H_{2}\right)$ hold; let $|f(x)| \geq|x|$ for all $|x| \geq x_{0}>0$ and let $k$ be a positive integer. For $p_{n} \equiv 0, m=2$, assume that

$$
0<\alpha \leq\left(\frac{k}{1+k}\right)^{1+k}
$$

if either

$$
\limsup _{n \rightarrow \infty} \sum_{i=n-k}^{n-1} q_{i}(i-k)>2\left(1-\frac{\alpha^{2}}{4}\right)
$$

or

$$
\limsup _{n \rightarrow \infty} \sum_{i=n-k}^{n-1} q_{i}(i-k)>2\left(1-\alpha^{k}\right)
$$

then (31) is oscillatory.

\section{Examples}

Example 1. Considering the equation

$$
\begin{aligned}
& \Delta^{m}\left(x_{n}+\frac{1}{n} x_{n-l}\right)+\frac{2^{(m-1)^{2}}(m-1) !\left[2+\left((-1)^{n} / n\right)\right]}{e(n-3)(n-2)^{m-2}} \\
& \quad \times x_{n-2} \ln \left(e+x_{n-2}^{2}\right)=0
\end{aligned}
$$

where $n>3, m$ is an even, and $l$ is a positive integer, then we have

$$
\begin{gathered}
0<p_{n}=\frac{1}{n}<1, \quad q_{n}=\frac{2^{(m-1)^{2}}(m-1) !\left[2+\left((-1)^{n} / n\right)\right]}{e(n-3)(n-2)^{m-2}}, \\
f(x)=x \ln \left(e+x^{2}\right), \quad k=2,
\end{gathered}
$$

where $\left\{q_{n}\right\}$ is a positive sequence. Then

$$
\begin{aligned}
& \sum_{i=n-2}^{n} q_{i}(i-2)^{m-1}\left(1-\frac{1}{i-2}\right) \\
& =\sum_{i=n-2}^{n} \frac{2^{(m-1)^{2}}(m-1) !\left[2+\left((-1)^{n} / n\right)\right]}{e} .
\end{aligned}
$$

Thus

$$
\begin{gathered}
\limsup _{n \rightarrow \infty} \sum_{i=n-2}^{n} \frac{2^{(m-1)^{2}}(m-1) !\left[2+\left((-1)^{n} / n\right)\right]}{e} \\
=\frac{6}{e} 2^{(m-1)^{2}}(m-1) !>2^{(m-1)^{2}}(m-1) ! .
\end{gathered}
$$

Therefore, by Theorem $8,(43)$ is oscillatory.

Example 2. Considering the equation

$$
\begin{aligned}
& \Delta^{m}\left[x_{n}+\frac{n-1}{n} x_{n-l}\right] \\
& \quad+\frac{2^{(m-1)^{2}}(m-1) !\left[(15 / 32)+\left((-1)^{n} / n\right)\right]}{(n-2)^{m-2}} \\
& \quad \times x_{n-2} \ln \left(e+x_{n-2}^{2}\right)=0,
\end{aligned}
$$


where $n>2, m$ is an even, and $l$ is a positive integer, then we have

$$
\begin{gathered}
0<p_{n}=\frac{n-1}{n}<1, \\
q_{n}=\frac{2^{(m-1)^{2}}(m-1) !\left[(15 / 32)+\left((-1)^{n} / n\right)\right]}{(n-2)^{m-2}}, \\
f(x)=x \ln \left(e+x^{2}\right), \quad k=2,
\end{gathered}
$$

where $\left\{q_{n}\right\}$ is a positive sequence. Denote $\alpha=4 / 14$; then

$$
\begin{aligned}
& \sum_{i=n-2}^{n-1} q_{i}(i-2)^{m-1}\left(1-\frac{i-2-1}{i-2}\right) \\
& =\sum_{i=n-2}^{n-1} 2^{(m-1)^{2}}(m-1) !\left[\frac{15}{32}+\frac{(-1)^{n}}{n}\right] .
\end{aligned}
$$

Thus

$$
\begin{aligned}
& \limsup _{n \rightarrow \infty} \sum_{i=n-2}^{n-1} 2^{(m-1)^{2}}(m-1) !\left[\frac{15}{32}+\frac{(-1)^{n}}{n}\right] \\
& \quad=\frac{15}{16} 2^{(m-1)^{2}}(m-1) !>2^{(m-1)^{2}}(m-1) !\left(1-\left(\frac{4}{14}\right)^{2}\right) .
\end{aligned}
$$

Therefore, by Theorem 11, (47) is oscillatory.

\section{Conflict of Interests}

The author declares that there is no conflict of interests regarding the publication of this paper.

\section{Acknowledgments}

The author sincerely thanks the reviewers for their valuable suggestions and useful comments that have led to the present improved version of the original paper. This work was supported by a Grant from the Natural Science Foundation of Shandong Province of China (no. ZR2013AM003) and the Development Program in Science and Technology of Shandong Province of China (no. 2010GWZ20401).

\section{References}

[1] R. P. Agarwal, Difference Equations and Inequalities, Marcel Dekker, New York, NY, USA, 2nd edition, 2000.

[2] I. Györi and G. Ladas, Oscillation Theory of Delay Differential Equations, Oxford Clarendon Press, Oxford, UK, 1991.

[3] Q. Zhang, J. Yan, and L. Gao, "Oscillation behavior of evenorder nonlinear neutral differential equations with variable coefficients," Computers and Mathematics with Applications, vol. 59, no. 1, pp. 426-430, 2010.

[4] I. P. Stavroulakis, "Oscillation criteria for first order delay difference equations," Mediterranean Journal of Mathematics, vol. 1, no. 2, pp. 231-240, 2004.
[5] E. Thandapani, R. Arul, and P. S. Raja, "Bounded oscillation of second order unstable neutral type difference equations," Journal of Applied Mathematics and Computing, vol. 16, no. 1-2, pp. 79-90, 2004.

[6] Z. Zhou, J. Yu, and G. Lei, "Oscillations for even-order neutral difference equations," Korean Journal of Computational \& Applied Mathematics, vol. 7, no. 3, pp. 601-610, 2000.

[7] S. Chen, "Oscillation criteria for certain even order quasilinear difference equations," Journal of Applied Mathematics and Computing, vol. 31, no. 1-2, pp. 495-506, 2009.

[8] E. Thandapani and R. Arul, "Oscillatory and asymptotic behavior of solutions of higher order damped nonlinear difference equations," Czechoslovak Mathematical Journal, vol. 49, no. 1, pp. 149-161, 1999.

[9] G. Ladas and C. Qian, "Comparison results and linearized oscillations for higher-order difference equations," International Journal of Mathematics and Mathematical Sciences, vol. 15, no. 1, pp. 129-142, 1992.

[10] L. Yang, "Oscillation behavior of even-order neutral difference equations with variable coefficients," Pure and Applied Mathematics, vol. 24, no. 4, pp. 796-801, 2008 (Chinese).

[11] G. Ladas, Ch. G. Philos, and Y. G. Sficas, "Sharp conditions for the oscillation of delay difference equations," Journal of Applied Mathematics and Simulation, vol. 2, no. 2, pp. 101-111, 1989.

[12] L. H. Erbe and B. G. Zhang, "Oscillation of discrete analogues of delay equations," Differential and Integral Equations, vol. 2, no. 3, pp. 300-309, 1989. 


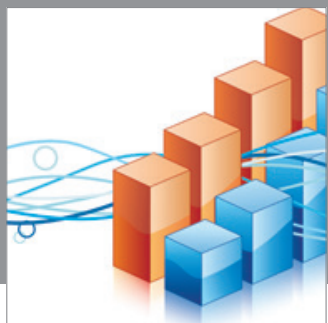

Advances in

Operations Research

mansans

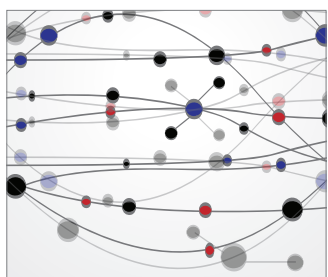

The Scientific World Journal
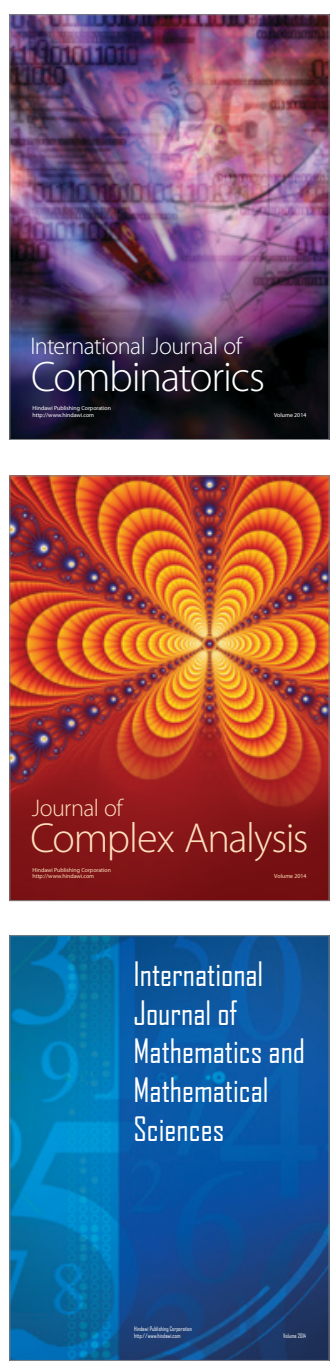
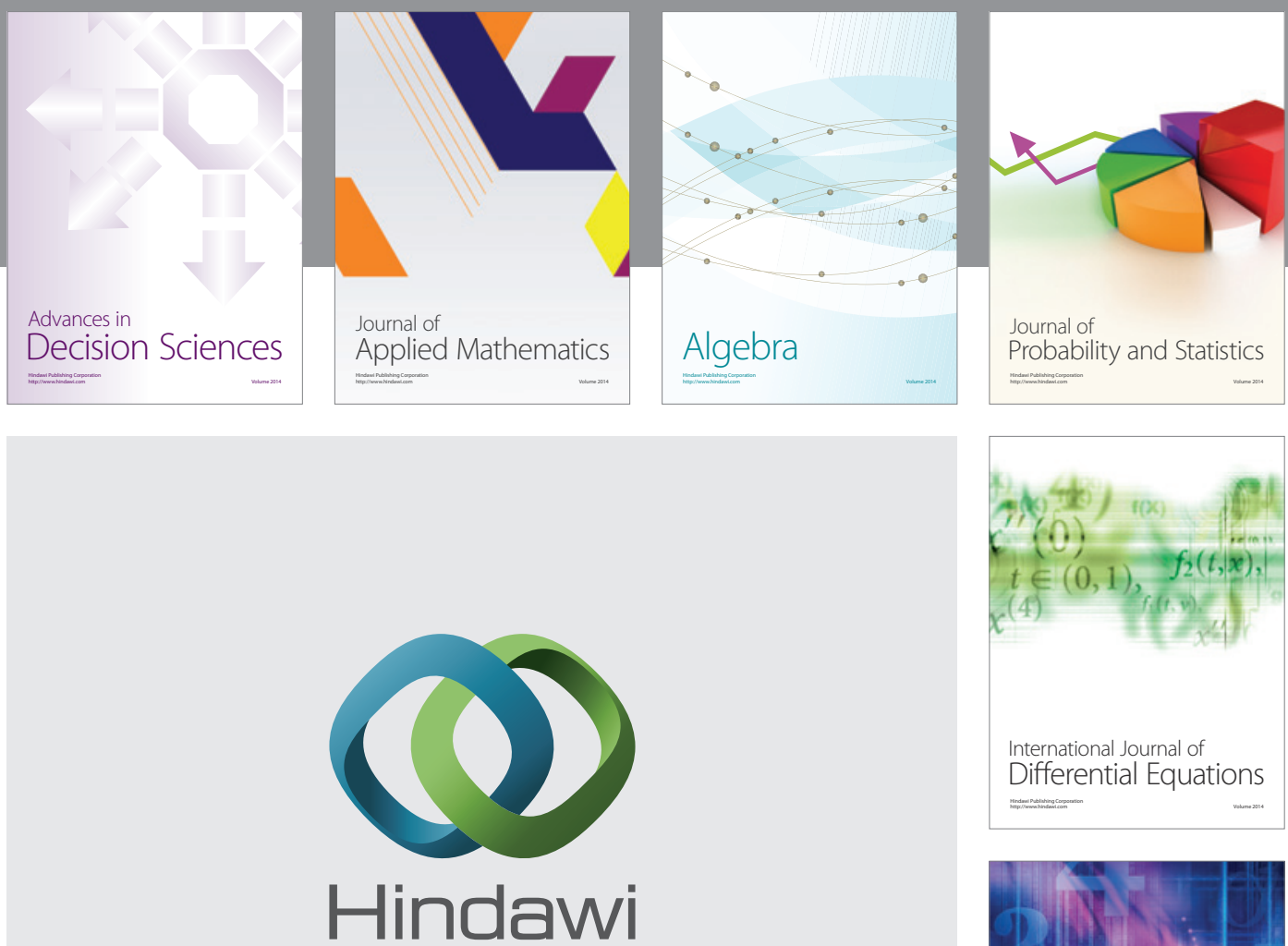

Submit your manuscripts at http://www.hindawi.com
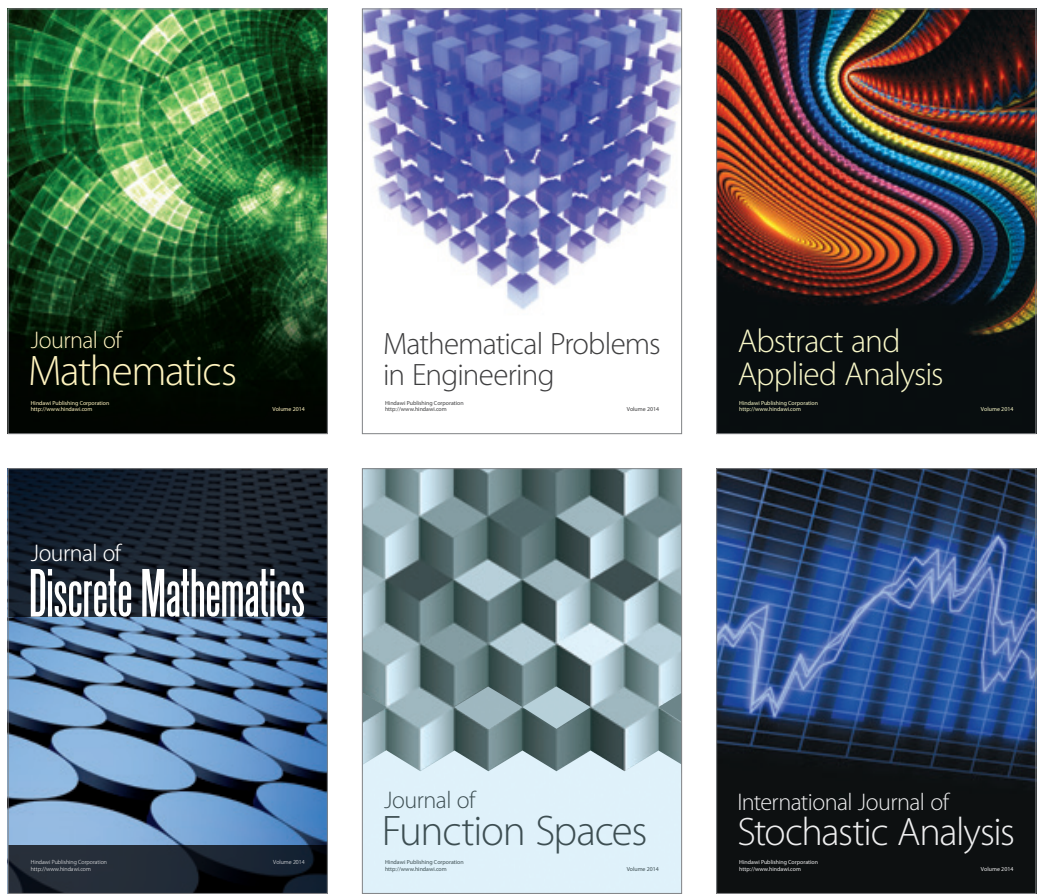

Journal of

Function Spaces

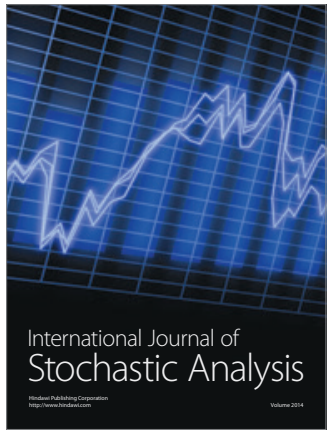

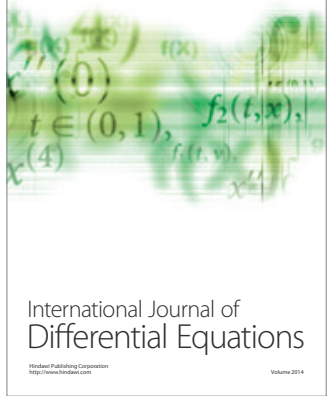
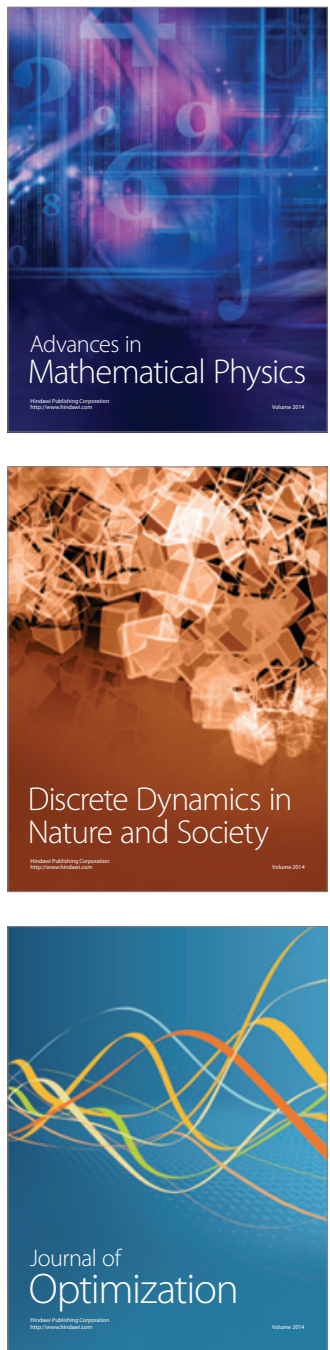